\title{
NEUROMARKETING:
}

\section{THE NEXT STEP IN MARKET RESEARCH?}

Christopher R Madan

Department of Psychology, University of Alberta

Eureka

Volume 1, Number 1 (2010)

\begin{abstract}
Neuromarketing is an emerging interdisciplinary field connecting psychology and neuroscience with economics. The goal of neuromarketing is to study how the brain is physiologically affected by advertising and marketing strategies. In order to evaluate the effectiveness of these strategies, brain activity resulting from viewing an advertisement is monitored and measured using neuroimaging techniques such as functional magnetic resonance imaging (fMRI) and electroencephalography (EEG). Neuromarketing studies usually measure preference between products in terms of brand familiarity or product preference. In traditional marketing studies, measures such as the product preference for a particular advertisement is sometimes difficult to measure, as a viewer may hold a cognitive bias. However, brand familiarity and product preference have been correlated with neural activity. The field of neuromarketing is still viewed with caution from consumer protection groups as well as many academics due to the possible ethical implications of designing advertisements to intentionally cause specific neurological effects.
\end{abstract}

\section{Introduction}

Neuromarketing is an emerging interdisciplinary field that combines psychology, neuroscience, and economics (Lee, Broderick, \& Chamberlain, 2007), with the term itself being coined just six years ago (Smidts, 2002). The goal of neuromarketing is to study how the brain is physiologically affected by advertising and marketing strategies. In order to evaluate the effectiveness of these strategies, brain activity resulting from viewing an advertisement is monitored and measured using neuroimaging techniques such as functional magnetic resonance imaging (fMRI) and electroencephalography (EEG) (Laybourne \& Lewis, 2005; Smidts, 2002). Neuromarketing studies usually measure preference between products in terms of brand familiarity or product preference. In traditional marketing studies, measures such as the product preference for a particular advertisement is sometimes difficult to measure, as a viewer may hold a cognitive bias. In neuromarketing studies, brand familiarity and product preference have been correlated with neural activity (McClure et al., 2004; Schaefer, Berens, Heinze, \& Rotte, 2006; Walter, Abler, Ciaramidaro, \& Erk, 2005).
The field of neuromarketing is viewed with caution from consumer protection groups as well as many academics due to the possible ethical implications of designing advertisements to intentionally cause specific neurological effects (Commercial Alert, 2003).

Intrinsic to neuromarketing are neuroimaging techniques - primarily functional magnetic resonance imaging (fMRI) and electroencephalography (EEG) - that comprise the neuroscience aspect of the field (Laybourne \& Lewis, 2005; Smidts, 2002). fMRI involves the participant lying on a bed, with their head positioned inside the large magnetic ring of a scanner. By monitoring the participant's brain with fMRI, researchers can measure the neural activity throughout the brain in terms of blood flow via oxygen usage. Contrasting this technique, EEG equipment is relatively light and portable. EEGs measure brain activity in terms of electrical activity at the scalp using numerous electrodes that are placed on the participant's scalp in a net-like fashion. Using neuroimaging, researchers can then conduct conditioning and marketing studies and monitor the participant's response, using both behavioural responses as 
well as neural activations. In layman's terms, Fugate (2007) describes neuromarketing to "managers and executives" as: "The process involves wiring subjects to various electronic devices and asking them to perform experimental tasks and control tasks. These devices generate instant, colorful images of a working brain and the researcher is able to compare differences in the images produced during the respective tasks. It then becomes apparent which parts of the brain have responded to the stimuli used." Fugate (2007) continues on to more subtle details of the mechanics behind neuromarketing, painting a picture of a revolution in the marketing field, however, some critical scientific concepts are overlooked by Fugate (2007), including the corollary nature to neuromarketing research.

Despite suggestions otherwise, 'neuromarketing' as a concept emerged prior to the word actually being coined in 2002 (Smidts, 2002). However, due to limitations of neuroimaging techniques conducted in the past few decades, many studies lacked the spatial resolution (ability to differentiate different regions of the brain) to make any useful claims as to the mechanisms behind effective and ineffective advertising techniques. For example, in an EEG study, Reeves, Lang, Thorson, and Rothschild (1989), claim that television scenes with positive messages cause greater left hemisphere activity in the frontal region, while negative content causes activation of the frontal portion of the right hemisphere. It is important to note that cortical arousal was only monitored in terms of frontal versus occipital, as only four electrodes were used (in addition to the two reference electrodes). Current EEG systems often have up to 256 electrodes to monitor brain activity, making them much more precise. Many other studies from the same time period also employ 'hemisphere' activations as key findings (i.e., Krugman, 1971; Rothschild, Hyun, Reeves, Thorson, \& Goldstein, 1988; Rothschild \& Hyun, 1990; Weinstein, Appel, \& Weinstein, 1980), including subtitles on papers reading broad statements such as, "Each medium had about the same effect on the left brain as on the right." (Weinstein et al., 1980). Nonetheless, it is not the fact that earlier 'neuromarketing' research has been imprecise that is of greatest importance, but rather how quickly the field has evolved over the last few years.

\section{Conditioning \& Marketing}

In neuromarketing research, two methods are typically employed as means of evaluating an individual's preference between products: brand familiarity and product preference.

\section{Brand Familiarity}

Brand familiarity involves comparisons between familiar and unfamiliar products. Campbell and Keller (2003) describe familiarity as habituating to a brand, as in mere exposure. When a consumer first sees an advertisement for an unfamiliar brand, they feel negative uncertainty towards it as it is unfamiliar. However, repetition of an advertisement message, at low levels, increases the effectiveness and decreases this uncertainty. One way that brands can become more familiar and earn the trust of the consumer is through the use of celebrity endorsements (Fugate, 2007). Advertisers must keep in mind not to advertise too much though, as the tedium of repeated exposures can decrease the effectiveness of the advertisement by annoying the viewer (Campbell \& Keller, 2003). For an already familiar product, repeated exposures provide more time for the consumer to process the advertisement and their associated experiences (i.e., experiences from using the product) as consumers can only store knowledge for the familiar, but not the unfamiliar. As such, for unfamiliar brands there is less knowledge to process, causing the consumer to become bored, and even annoyed, more easily. In essence, new brands just breaking into the market need to be conservative in their marketing efforts in so as to not overdo it, but also enough to cause consumers to recognize their brand. More seasoned brands (i.e., Coca-Cola) are able to advertise more often 
with less concern of annoying their audience (Campbell \& Keller, 2003).

\section{Product Preference}

Unlike brand familiarity, product preference comparisons involve two known brands or products. For example, Walter et al. (2005) asked male participants to rate a car's attractiveness irrespective of cost and practical considerations, given the choice between a sports car, a medium-sized car, and a small car. Participants ranked the sports car first, followed by the medium-sized car, with the small car ranked last. Walter et al. (2005) described sports cars as a primary reinforcer for social dominance, representing speed, power, and independence. In this instance, the sports car acted as a secondary reward. Secondary rewards are described as items such as cultural goods or money that reinforce behaviour only after prior learning, through associations with primary rewards (innate reinforcers including food, water, and sexual stimuli). Walter et al. (2005) also outlined three main functions of rewards. Rewards can: (a) induce learning via positive reinforcement, (b) induce consuming behaviour for acquiring the reward, and (c) induce positive affect. As seen from Walter et al.'s study (2005) sports cars are preferred as they represents characteristics that our culture values, and that we believe to be correlated with primary rewards that we innately seek. This study was also adapted from a previous study of social hierarchy and dominance involving monkeys (Morgan et al., 2002, as cited by Walter et al., 2005). In summary, given two known products, preference for one over the other is due primarily to the preferred product exhibiting more reinforcing qualities in terms of secondary reinforcers relevant to us personally, as well as to our cultural background.

\section{Neural Correlates}

Neuromarketing, is based on finding a neural correlates for buying behaviours such as brand familiarity and product preference. It is important to acknowledge that researchers are only able to seek a 'correlate' as most studies are only able to monitor neural activity observationally, and do not induce product preference via neural stimulation. With this in mind, compelling evidence has been found linking the medial prefrontal cortex with both brand familiarity and product preference. Damasio (1996) implicates the medial prefrontal cortex (mPFC) as a "repository of linkages between factual knowledge and bioregulatory states". In the more specific instance of advertising, this translates into product information and experiences being linked to positive affect, via the mPFC.

Studies that point to the medial prefrontal cortex (mPFC) as the locus of interest for neuromarketing studies are quite notable. Kable and Glimcher (2007) have also correlated mPFC activity, along with several other regions, directly with subject preference. Similarly, product preference (in the 'sports car' study outlined earlier) has been correlated with the activation of several brain regions in the reward circuitry of the brain, including the mPFC (Walter et al., 2005). Preference has also been correlated with mPFC activity independent of prices, and was found to be predictive of subsequent purchasing (Knutson, Rick, Wimmer, Prelec, \& Loewenstein, 2007). A simpler preference judgement study was also conducted prior to the aforementioned studies by (Paulus \& Frank, 2003), using a visual discrimination task as a control and found coinciding results.

One of the most compelling neuromarketing studies was that conducted by McClure et al. (2004). In the study, the researchers monitored neural activity when drinking either Coca-Cola or Pepsi. McClure et al. (2004) used an fMRI experiment with two conditions, (a) blind taste test and (b) brand-cued delivery. With blind taste test, brain activity between the Coca-Cola and Pepsi was nearly identical. However, in the brand-cued condition, dramatic differences were found in neural activity, primarily in the ventromedial prefrontal cortex. The important finding was that no neural activation differences were found when no brand information was 
available, but when brands were known, brand familiarity and product preference come into play and Coca-Cola was found to be generally preferred in the participants and caused significantly more activity in the ventromedial prefrontal cortex. One key aspect of the study is that no choices are actually made by the participant; the drink was delivered directly to the participant in the fMRI in small quantities, the manipulation was based on if the brand was announced first, and the finding is based on the resulting activated regions as measured by the fMRI. Previous conditioning and brand preference is only demonstrated in the brandcued delivery, and only then is there significant ventromedial prefrontal cortex activation.

A follow-up to the McClure et al. (2004) study sheds more light on the paradox of cola preference (Koenigs \& Tranel, 2008). Koenigs and Tranel (2008) explain that there is a "Pepsi paradox", which basically explains that in a blind-taste test, subjects tend to prefer Pepsi over Coca-Cola, or have no reliable preference, yet Coca-Cola consistently outsells Pepsi. The paradox is that when brand information is available, Coca-Cola is preferred, however, when brand information is not provided, no reliable preferences can be made. In the McClure et al. (2004) study, cola preference was counterbalanced. Koenigs and Tranel (2008) confirm predictions from previous correlative data by using subjects with prefrontal cortex damage, finding that even when these patients are presented with brand information, it makes no difference on their preferences. Thus this finding mirrors effects found in normal individuals in blind-taste tests, as well as in their own blindtaste test condition. Explained elegantly by Montague (2006), McClure et al. (2004) used a design very similar to Pavlov's original classical conditioning studies, however, they were not interested in a subject's brain response to each drink, but rather the responses that predicted subject's choices prior to being in the MRI scanner. McClure et al. (2004) were not interested specifically in the choices between the two colas, but rather in the valuation of the two choices, prior to the decision itself. Taste itself is not the reason Coca-Cola is preferred over Pepsi; rather it is the strong brand image of Coca-Cola (Gladwell, 2005).

Brand familiarity has also been connected with the mPFC in several studies. Differences in neural activity when comparing familiar and unfamiliar products has been associated with mPFC activity (Schaefer et al., 2006, Schaefer \& Rotte, 2007), which can also be connected to 'neurolearning' literature of novelty detection in rat lesion studies (Dias \& Honey, 2002). Relative to behavioural principles, Campbell and Keller (2003) suggest that brand familiarity is of extreme importance to advertisers. Consumers tend to fear the unknown, and in advertising, this fear materializes in an uncertainty of the product and instead they choose a known analogous product. Schaefer and Rotte (2007) demonstrate this as increased $\mathrm{mPFC}$ and superior frontal gyri activity for culturally familiar brands relative to unfamiliar brands.

In summary, medial prefrontal cortex (mPFC) activation has been linked to preference judgements by many studies (McClure et al., 2004; Paulus \& Frank, 2003; Walter et al., 2005). In addition, 'preference' for the familiar over the unfamiliar can also be attributed to the mPFC (Schaefer et al., 2006; Schaefer \& Rotte, 2007). Assuming that the consumer is going to buy a product either way (i.e., a car), preferences between the available choices in terms of their relative value is the next step in the decision making proces (Montague, 2008). By weighing the pros and cons of all the available choices, consumers can evaluate their choices. Research indicates that this process is undertaken primarily by the medial prefrontal cortex, which some have dubbed the 'liking centre' of the brain (Sutherland, 2004).

Other than the medial prefrontal cortex, several other areas have been implicated as key brain regions relevant to neuromarketing research (Walter et al., 2005). Some of these regions include the amygdala, ventral striatum, and 
orbitofrontal cortex. The amygdala is commonly known for its role in processing emotional information, however, it has also been correlated with reward intensity in neuromarketing studies (Walter et al., 2005). The ventral striatum, which includes the nucleus accumbens - the reward center of the brain, has been correlated with selfreported self arousal (salience) but only as an indicator of the predicted value of the reward (Knutson et al., 2007; Walter et al., 2005). This is thought of as 'prediction error' and is used as a mechanism for learning. The orbitofrontal cortex (OFC) is mainly thought of as a measure of preference, and consists of mainly two regions: the medial and lateral (Walter et al., 2005). The medial OFC, which includes the medial prefrontal cortex, is activated by rewarding stimuli. Lateral OFC activity is correlated with punishing stimuli. However, the use of neuroimaging techniques is not solely limited to neural activation measures. For example, neuroimaging quantitatively measure affect in terms of hormonal secretions such as dopamine (Fugate, 2007). Even still, there is much to discover in terms of neural correlates of interest to neuromarketing, though the field is expanding rapidly.

\section{Ethics of Neuromarketing}

A major issue for research in neuromarketing is the ethical concerns of neuroimaging in order to enhance commercial gain. From a scientific standpoint, neuromarketing is nowhere near being able to allow researchers to design a marketing campaign so addictive that overrides an individual's free will, though concerns are being allayed regarding this, founded or unfounded. In the United States, a consumer protection group, Consumer Alert, has filed complaints to universities, the US federal government, as well as a US senate committee, protesting the ethics of neuromarketing. They describing neuromarketing as "find[ing] a buy button inside the skull" (Commercial Alert, 2003). Commercial Alert (2003) claims: "Our children are suffering from extraordinary levels of obesity, type 2 diabetes, anorexia, bulimia, and pathological gambling, while millions will eventually die from the marketing of tobacco." According to Consumer Alert (2003), the rise of neuromarketing will bring an end to free will.

Many academics are also hesitant to embrace the field, Lee et al. (2007) posits: "Unfortunately, the barely concealed disdain for the idea of 'neuromarketing' in the neuroscience literature is clearly based on the opinion that marketing research is a commercial activity purely designed to sell products to the public...” Though there are many journals dedicated to economics and marketing, key aspects of neuromarketing, neuroscience academics tend to focus on more medically relevant, or abstract, questions (Thompson, 2003). As such, some believe that "brain imaging will be used in ways that infringe personal privacy to a totally unacceptable degree" (Editorial, 2004b). A similar stance was also made by an anonymous author in Nature Neuroscience, "Neuromarketing is little more than a new fad exploited by scientists and marketing consultants to blind corporate clients with science.” (Editorial, 2004a; Laybourne \& Lewis, 2005).

Research into neuromarketing may actually help reduce the problems raised by Commercial Alert (2003). For example, by examining the differences between the brain activity of compulsive overpurchasers relative to those who maintain more appropriate levels of purchasing may help us understand why these compulsive individuals tend to spend outside of their means. In addition, correlations between buying behaviour and clinical disorders can provide useful information for how clinicians can treat these disorders. For example, the medial prefrontal cortex and nucleus accumbens are quite important in value-based decision-making and the reward circuitry of the brain. These areas have also been implicated as areas relevant in schizophrenia (Montague, Hyman, \& Cohen, 2004).

Murphy, Illes, and Reiner (2008) believe that two major ethical issues are present in 
neuromarketing research: (a) protecting vulnerable parties from harm, and (b) protection of consumer autonomy if neuromarketing reaches critical effectiveness. Murphy et al. (2008) also outline recommendations for a 'code of ethics' to be adopted by the neuromarketing industry. Some of their recommendations include protecting research subjects from coercion, full disclosure of ethical principles used in the study, and accurate representation of scientific methods to businesses and the media.

\section{Free will \& Decision-making}

However, if neuromarketing ever does reach critical effectiveness as Murphy et al. (2008) suggests, the concerns of Commercial Alert (2003) may not be unfounded after all, and neuromarketing may come to infringe on an individual's free will. The significance of neuromarketing is not confined to neuroimaging techniques, but also encompasses computational neuroscience, the study of quantifying the component steps that underlie a given behavioural process. For example, value-based decision-making can be broken down into five steps: (a) identifying the decision problem; (b) weighing the possible choices; (c) making a decision based upon the evaluation of the choices available; (d) after carrying out the decision, consider the resulting consequences; and (e) learn from the decision-making process in order to make better decisions in the future (Rangel, Camerer, \& Montague, 2008). "Viewed this way, it's easy to see why 'free' choice is an unconstructive way to conceptualize the way humans choose...” (Montague, 2008). Free will, and the ability to manipulate an individual's perception of it have also recently come to light (Vohs \& Schooler, 2008). However, it has been many years since neuroimaging studies have suggested that neural activity does precede conscious intention, calling the issue of free will into question, especially if it can be monitored by an outside observer (Libet, Gleason, Wright, \& Pearl, 1983). Welberg (2007) describes that in the context of neuromarketing, Knutson et al. (2007) showed that the decision of whether to buy a product or not results from a balancing act of the gain of obtaining the product, offset by the downside of actually having to pay for the product - in essence an interplay of choices and the corresponding valuations.

Walvis (2008) further connects neuroscience with common marketing principles using computational neuroscience, rather than neuroimaging. Walvis (2008) suggests three propositions of neural cortical representation, or how the brain organizes information. These three propositions function similarly to the basis of an artificial neural network model, implicating the importance of what other 'elements' the brand is associated with, the strength of these associations, and the sheer number of associations that are present between the brand and other 'elements' in the network (Walvis, 2008). These correlate into "Three Branding Laws", based upon how personally relevant the brand's marketing strategy is to the consumer, how repetitive and targeted the branding efforts are, and how engaging the branding environment is to the consumer. The stronger these connections and 'pathways' are, the more likely a given brand will be chosen by the consumer. By using these 'laws', we can again quantify factors involved in choice behaviour, through the use of an artificial neural network With these techniques we not only can observe the interacting elements intrinsic to decision-making in terms of neural activations, but even to a more basic level, though the weightings of different choice options does start to become conjecture. Nonetheless, factors such as choice assortment and notions of an ideal point can be examined, weighing each product's valuations with the Hebbian-like theories presented by Walvis (2008). Using a strong neuroscientific basis for a branding, as suggested by Walvis (2008), neuromarketing can greatly improve marketing techniques, even without the use of neuroimaging, but rather employing other aspects of neuroscience. 


\section{Conclusion}

Fugate (2007) suggests several key implications of neuromarketing that suggest a revolution will soon overcome current market research. Using neuromarketing, researchers can evaluate an advertisement's effectiveness much more scientifically, in terms of the viewer's attention to the ad, as well as how the ad affects the viewer's emotional state (i.e., excitement or humour). Product appeal, and the 'sports car' study (Walter et al., 2005) are also implicated regarding their findings with the reward circuitry of the brain. Fugate (2007) continues on to celebrity endorsements and how neuromarketing can connect and quantify the effects of the auditory and visual stimuli of the celebrity as they translate into hormonal secretions which lead to a positive emotional response and feelings of trust. Also important is logo/brand selection, as researched by McClure et al. (2004) with their findings involving brand recognition and emotional attachment, with the result that CocaCola outperforms Pepsi. The future implications of neuromarketing show great potential and only time will tell how much of an effect these new techniques will have on marketing success.

At its current stage, neuromarketing is by no means adequate in determining if an advertisement is effective. Simply causing activation in regions such as the medial prefrontal cortex does not necessarily indicate that an advertisement is effective as it is only a corollary measure. Also, the region is the focus of many other research studies including those in fear conditioning (Baratta, Lucero, Amat, Watkins, \& Maier, 2008), eating disorders provocation (Uher et al., 2004), and startle responses (Day-Wilson, Jones, Southam, Cilia, \& Totterdell, 2006). Despite the current flaws in neuromarketing research, the field shows great promise as being the next step in market research. If advertisers took advantage to the many psychology studies that have been previously conducted, they would undoubtedly be more successful in both making a longer lasting impression on the consumer, as well as being able to better direct their efforts towards a target demographic. By adding the techniques of neuroimaging to their arsenal, in the form of neuromarketing, advertising agencies could perform much better. If improved marketing capabilities is good or bad for the consumer is quite debatable, but with proficient ethics being enforced upon the field (as they are with most other scientific research disciplines) only good can come from the pairing of the commercial principles of economics with the learning principles of psychology and neuroscience.

\section{References}

Baratta, M. V., Lucero, T. R., Amat, J., Watkins, L. R., \& Maier, S. F. (2008). Role of the ventral medial prefrontal cortex in mediating behavioral control-induced reduction of later conditioned fear. Learning \& Memory, 15(2), 84-87.

Campbell, M., \& Keller, K. L. (2003). Brand familiarity and ad repetition effects. Journal of Consumer Research, 30, 292-304.

Commercial Alert. (2003, Dec 1). Commercial alert asks emory university to halt neuromarketing experiments. Commercial Alert News Release. retrieved october 15, 2008, from the world wide web: http://www.commercialalert.org/PDFs/neuromarketin grel.pdf.

Damasio, A. R. (1996). The somatic marker hypothesis and the possible functions of the prefrontal cortex. Philosophical transactions of the Royal Society of London, Series B, Biological sciences, 351, 1413-1420.

Day-Wilson, K. M., Jones, D. N. C., Southam, E., Cilia, J., \& Totterdell, S. (2006). Medial prefrontal cortex volume loss in rats with isolation rearinginduced deficits in prepulse inhibition of acoustic startle. Neuroscience, 141(3), 1113-1121.

Dias, R., \& Honey, R. C. (2002). Involvement of the rat medial prefrontal cortex in novelty detection. Behavioral Neuroscience, 116(3), 498-503.

Editorial. (2004a). Brain scam? Nature Neuroscience, 7(10), 1015.

Editorial. (2004b). Neuromarketing: beyond branding. The Lancet Neurology, 3, 71. 
Fugate, D. L. (2007). Neuromarketing: a layman’s look at neuroscience and its potential application to marketing practice. Journal of Consumer Marketing, 24(7), 385-394.

Gladwell, M. (2005). Blink. New York: Time Warner Book Group.

Kable, J. W., \& Glimcher, P. W. (2007). The neural correlates of subjective value during intertemporal choice. Nature Neuroscience, 10(12), 1625-1633.

Knutson, B., Rick, S., Wimmer, G. E., Prelec, D., \& Loewenstein, G. (2007). Neural predictors of purchases. Neuron, 53, 147-157.

Koenigs, M., \& Tranel, D. (2008). Prefrontal cortex damage abolishes brand-cued changes in cola preference. Social Cognitive \& Affective Neuroscience, 3(1), 1-6.

Krugman, H. E. (1971). Brain wave measures of media involvement. Journal of Advertising Research, 11, 3-9.

Laybourne, P., \& Lewis, D. (2005). Neuromarketing: the future of consumer research? Admap, 461, 28-30.

Lee, N., Broderick, A. J., \& Chamberlain, L. (2007). What is 'neuromarketing'? A discussion and agenda for future research. International Journal of Psychophysiology, 63, 199-204.

Libet, B., Gleason, C. A., Wright, E. W., \& Pearl, D. K. (1983). Time of conscious intention to act in relation to onset of cerebral activity (readinesspotential). the unconscious initiation of a freely voluntary act. Brain, 106(Pt 3), 623-642.

McClure, S. M., Li, J., Tomlin, D., Cypert, K. S., Montague, L. M., \& Montague, P. R. (2004). Neural correlates of behavioral preference for culturally familiar drinks. Neuron, 44, 379-387.

Montague, R. (2006). Why choose this book? : How we make decisions. Toronto: Penguin Group.

Montague, R. (2008). Free will. Current Biology, 18(4), R584-R585.

Montague, R., Hyman, S. E., \& Cohen, J. D. (2004). Computational roles for dopamine in behavioural control. Nature, 431, 760-767.

Murphy, E., Illes, J., \& Reiner, P. B. (2008). Neuroethics of neuromarketing. Journal of Consumer Behavior, 7, 293-302.
Paulus, M. P., \& Frank, L. R. (2003). Ventromedial prefrontal cortex activation is critical for preference judgments. Neuroreport, 14, 1311-1315.

Rangel, A., Camerer, C., \& Montague, P. R. (2008). A framework for studying the neurobiology of valuebased decision making. Nature Reviews Neuroscience, 9(7), 545-556.

Reeves, B., Lang, A., Thorson, E., \& Rothschild, M. (1989). Emotional television scenes and hemispheric specialization. Human Communication Research, 15(4), 493-508.

Rothschild, M., \& Hyun, Y. J. (1990). Predicting memory for components of TV commercials from EEG. Journal of Consumer Research, 16, 472-478.

Rothschild, M., Hyun, Y. J., Reeves, B., Thorson, E., \& Goldstein, R. (1988). Hemispherically lateralized EEG as a response to television commercials. Journal of Consumer Research, 15, 185-198.

Schaefer, M., Berens, H., Heinze, H., \& Rotte, M. (2006). Neural correlates of culturally familiar brands of car manufacturers. Neuroimage, 31, 861-865.

Schaefer, M., \& Rotte, M. (2007). Favorite brands as cultural objects modulate reward circuit. Neuroreport, 18, 141-145.

Smidts, A. (2002). Kijken in het brein: Over de mogelijkheden van neuromarketing. (Brain imaging: Opportunities for neuromarketing). Inaugural Address Erasmus University: ERIM EIA-12-MKT.

Sutherland, M. (2004, July 24). Synopsis of reported neuromarketing studies. Retrieved October 12, 2008, from the World Wide Web:

http://www.sutherlandsurvey.com/Columns_Papers/S ynopsis $\backslash \% 20$ of $\ \% 20$ Neuromarketing $\backslash \% 20$ Studies.pdf.

Thompson, C. (2003, Oct 26). There's a sucker born in every medial prefrontal cortex. New York Times. Retrieved November 8, 2008, from the World Wide Web:

http://www.nytimes.com/2003/10/26/magazine/26BR AINS.html.

Uher, R., Murphy, T., Brammer, M. J., Dalgleish, T., Phillips, M. L., Ng, V. W. (2004). Medial Prefrontal Cortex Activity Associated With Symptom Provocation in Eating Disorders. American Journal of Psychiatry, 161(7), 1238-1246. 
Vohs, K. D., \& Schooler, J. W. (2008). The value of believing in free will: Encouraging a belief in determinism increases cheating. Psychological Science, 19(6), 49-54.

Walter, H., Abler, B., Ciaramidaro, A., \& Erk, S. (2005). Motivating forces of human actions:

Neuroimaging reward and social interaction. Brain Research Bulletin, 67, 368-381.
Walvis, T. H. (2008). Three laws of branding: Neuroscientific foundations of effective brand building. Journal of Brand Management, 16, 176-194.

Weinstein, S., Appel, V., \& Weinstein, C. (1980). Brain-activity responses to magazine and television advertising. Journal of Advertising Research, 20(3), 57-63.

Welberg, L. (2007). Shopping centres in the brain. Nature Reviews Neuroscience, 8(2), 84-85.

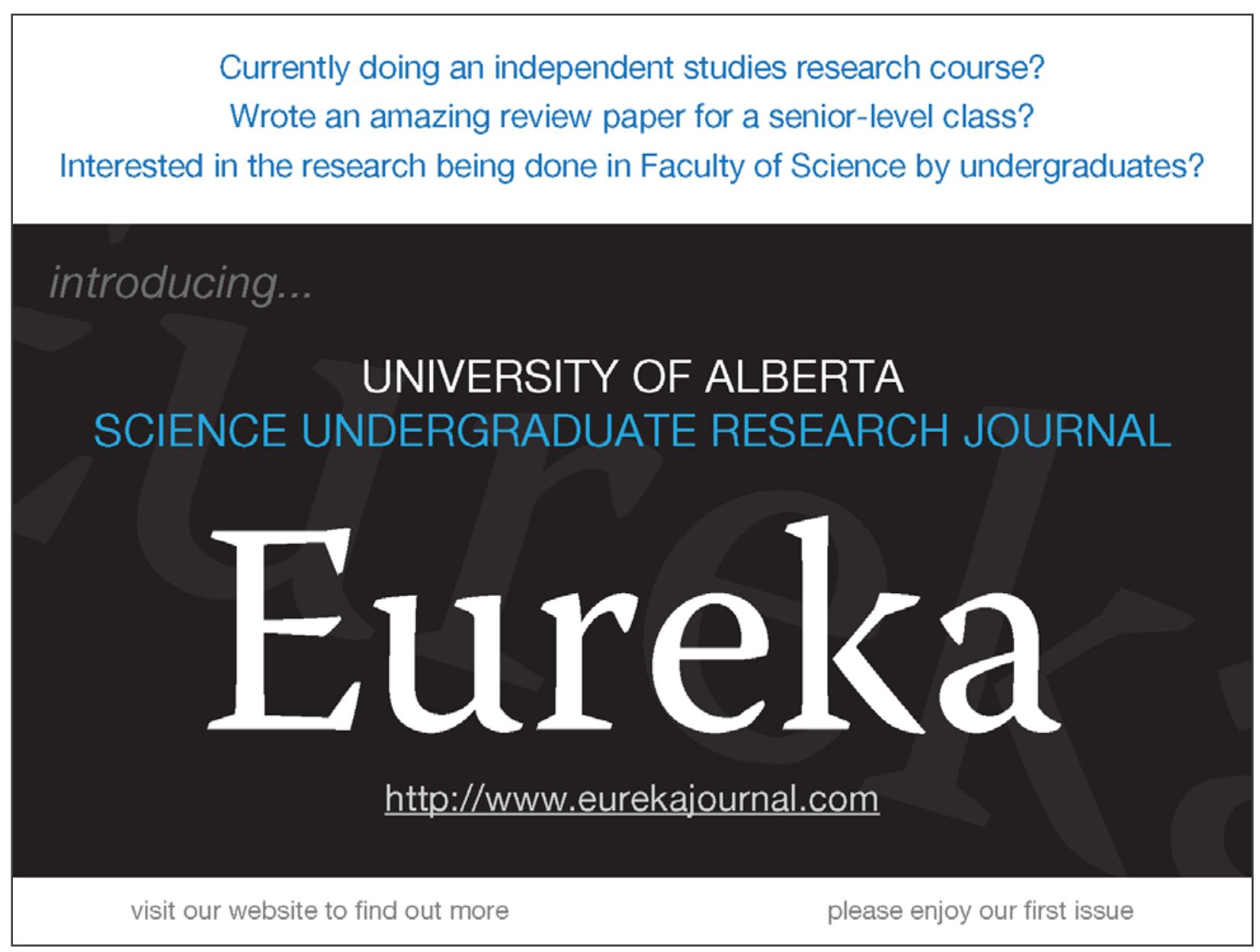

